\title{
Evaluation of micromorphological changes in tooth enamel after mechanical and ultrafast laser preparation of surface cavities
}

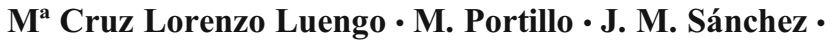 \\ M. Peix • P. Moreno • A. García • J. Montero • \\ A. Albaladejo
}

Received: 17 November 2011 / Accepted: 21 June 2012 / Published online: 4 July 2012

(C) Springer-Verlag London Ltd 2012

\begin{abstract}
The aim of this in vitro study was to evaluate the morphological changes that occur in tooth enamel after mechanical instrumentation and after femtosecond laser irradiation with different parameters via light and scanning electron microscopy (SEM). Twelve totally impacted third molars were collected and sectioned to provide several cut surfaces. These surfaces were exposed to infrared $(\lambda=795 \mathrm{~nm}, 120 \mathrm{fs}, 1-\mathrm{kHz}$ repetition rate, maximum mean power $1 \mathrm{~W}$ ) laser pulses and machined by means of a conventional mechanical technique. Two very different geometrical patterns were performed with femtosecond laser pulses: shallow rectangular cavities and deep cylindrical ones. The results of both machining procedures were examined using light and scanning electron microscopy. The SEM images show the femtosecond laser ability to produce high-precision cavities in tooth enamel. No signs of collateral damage, burning, melting, or cracks were observed despite the far different laser pulse energies used (ranging from 7 to $400 \mu \mathrm{J}$ ), unlike what is seen with conventional mechanical techniques. The femtosecond laser has the potential to become
\end{abstract}

M. C. L. Luengo $(\bowtie) \cdot$ M. Portillo $\cdot$ M. Peix $\cdot$ J. Montero

A. Albaladejo

Faculty of Medicine, Dental Clinic, University of Salamanca,

Prolongación del Paseo de la Universidad de Coimbra S/N,

37007 Salamanca, Spain

e-mail: mcruzlorenzo@hotmail.com

A. Albaladejo

e-mail: albertoalbaladejo@hotmail.com

P. Moreno • A. García

Laser Microprocessing Research Group, University of Salamanca,

Plaza de la Merced S/N,

37008 Salamanca, Spain

J. M. Sánchez

Department of General and Atmospheric Physics, Sciences

Faculty, Building Trilingual, University of Salamanca,

Plaza de la Merced S/N,

37008 Salamanca, Spain an optimal tool for the treatment of dental decay and as an alternative to the conventional drill to reduce mechanical damage during removal of the hard dental tissue.

Keywords Femtosecond $\cdot$ Laser $\cdot$ Enamel $\cdot$ Morphological alterations

\section{Introduction}

Restorative dentistry focuses on the development of new methods for caries removal and cavity preparation, as well as new restorative materials to ensure that treated teeth are restored, aesthetically and functionally with great precision [1]. When making cavities and removing carious tissue, the conventional mechanical technique is not as accurate as desired and has certain drawbacks, such as the nonselective removal of hard dental tissue, pain, and the need for anesthesia, among others [2]. This has gradually given way to new dental techniques for the removal of material as an alternative to conventional mechanical procedures [3-5].

Among these methods, high-intensity lasers have been widely used and approved by professionals [6]. Indeed, in the future, lasers may replace dentists' drills so that a more selective removal of material can be achieved [7-9]. In recent years, ultrashort-pulsed lasers have proved to be a very promising tool owing to their ability to avoid the formation of microcracks around treated areas, since such cracks can evidently lead to the formation of a new caries [10].

In laser processing, pulse duration is a crucial parameter. This parameter determines the mechanism of interaction between the light emitted and the tissue [11]. In recent years, laser sources based on titanium/sapphire crystals (Ti/S) [12], which produce extremely short pulses below the picosecond $\left(10^{-12} \mathrm{~s}\right)$ scale, have been developed. These laser pulses, amplified up to energies of the order of millijoules and 
conveniently focused on the surface of materials, allow the ablation of these surfaces with extreme precision and reproducibility, and cause much less collateral damage to the adjacent material than any other thermal, chemical, or mechanical process $[12,13]$.

In brief, ultrafast ablation is based on the non-linear processes of light absorption and ionization unleashed by the effect of irradiation with very short and intense pulses [14]. During the pulse, a thin layer on the surface of the tissue is almost fully ionized, giving rise to a dense electronic plasma able to break the bonds between ions or molecules as a result of the intense electric field. These species leave the surface together with the electrons in a process known as Coulomb explosion, which is a completely non-thermal process [15]. The effects derived from thermal load on the surrounding areas are therefore nonexistent. If the intensity is very high, the electronic plasma may absorb further energy, which is then radiated outwards, leading to a sudden increase in the temperature of a deeper layer, in turn resulting in the violent expulsion of both vapor and liquid droplets in equilibrium. This process, called phase explosion, is a thermal phenomenon, but it takes place fast enough to limit the diffusion of heat to very few microns of the surrounding tissue $[16,17]$. Thus, the irreversible damage to dental pulp tissue-which is particularly sensitive to such thermal effects, as well as microfractures or "cracks" in hard dental tissues produced by conventional laser sources - becomes almost negligible.

The aim of this in vitro study was to evaluate with light and scanning electron microscopy (SEM) microscopy the morphological changes that occur in enamel after mechanical instrumentation and after femtosecond laser irradiation with different parameters.

\section{Materials and methods}

\section{Collection and sample preparation}

Twelve totally impacted third molars were collected; the pieces had been extracted over a period of one week at the Oral and Maxillofacial Surgery Unit of the Virgen de la Vega Hospital in Salamanca.

The third molar was extracted from patients aged between 20 and 25. The molars were kept in an appropriate environment (physiological saline solution at $37^{\circ} \mathrm{C}$ ) to avoid dryness until the time of preparation. Each tooth was cross-sectioned transversely into two parts: the crown and the level of the root line enamel. The coronal part of the tooth was cut longitudinally in the vestibulo-lingual direction, in sheets of approximately 1-mm thickness using a precision Accutom-50 cutter/grinder (Struers, Denmark, Copenhagen) and 330K Struers diamond blades under a water coolant. One-millimeter-thick serial sections were taken at 3,200 rpm, with a driving speed of $0.460 \mathrm{~mm} / \mathrm{s}$ and high strength in order to obtain three to four slices from each specimen. The sheets containing only dentin were discarded. The samples were ground with sandpaper granulated at 300,400 , and 600 in a polishing machine.

To perform the experiments, each sample was divided in two groups according to dental cavity preparation:

- First group: mechanical cavity preparation.

- Second group: laser cavity preparation.

Each laser sample was treated using all the parameters investigated.

The samples were then preserved in physiological saline solution in an oven at $37{ }^{\circ} \mathrm{C}$ (for a maximum period of 1 month) to prevent them from drying out until the microscopy studies were performed.

\section{First group: mechanical dental cavity preparation}

For traditional dental cavity preparation a Supertorque Kavo Lux 655B handpiece was used at a speed of $450,000 \mathrm{rpm}$, with a tungsten round bur of $0.5 \mathrm{~mm}$ (ref. H1314005 Lemgo, Germany), with a water coolant. These were made with perforations of about $500-600 \mu \mathrm{m}$ in diameter in the enamel samples.

\section{Second group: dental cavity preparation by laser}

The microstructuring of the samples was performed with a system consisting of a Ti/sapphire oscillator (Spectra Physics, Tsunami) and a regenerative amplifier (Spectra Physics, Spitfire), based on the CPA technique [18], which produces pulses of $120 \mathrm{fs}\left(1 \mathrm{fs}=10^{-15} \mathrm{~s}\right)$; a wavelength in the near infrared region (795 nm), and energies up to $1 \mathrm{~mJ}$, with a repetition rate of $1 \mathrm{kHz}$ (mean power=1 W). The pulses were focused perpendicularly onto the sample surface by means of an achromat doublet, with a focal length of $100 \mathrm{~mm}$.

The samples were placed on an XYZ stage (Nanotec Micos ES100) in order to provide a precise location of the cavity and to properly focus the laser pulses on the surface. Two kinds of microstructured samples were prepared: shallow rectangular cavities and deep cylindrical ones. Since the size of these structures was required to be larger than the spot size, different strategies were adopted. For the rectangular structures, we scanned the surface by moving the samples following a meandering path with different pitches or distance between neighboring laser scans $(0.02$ and $0.01 \mathrm{~mm}$ ). Pulse energies of $0.007,0.01,0.03$, and $0.05 \mathrm{~mJ}$ were used to induce shallow structures; for each energy, cavities were made with different scanning speeds $(0.05$ and $0.1 \mathrm{~mm} / \mathrm{s})$. 
To achieve large (diameters up to $500 \mu \mathrm{m}$ ) and deep (up to $400 \mu \mathrm{m}$ ) cylindrical cavities, a helical drilling optics system (TGSW, Stuttgart) was used. This is an optomechanical device consisting of a number of optical wedges which can rotate (up to $3,000 \mathrm{rpm}$ ) and whose relative position along the rotating axis may be modified in order to force the laser spot to follow an eccentric path of variable diameter and at the same time to provide oblique incidence of the laser beam on the surface of the sample. Both features allow, on one hand, to drill microstructures with dimensions much larger than the laser spot size and, on the other hand, to overcome the depth limitations and tapering resulting of light propagation within the ablation crater (saturation effects) [19]. Since the number of free parameters became too large, we have always used the same rotating speed for the helical drilling system $(250 \mathrm{rpm})$ to fix the number of pulses irradiating a unit of area on the surface and the maximum beam inclination provided by the system $\left(4^{\circ}\right)$ to get the best approach to cylindrical geometry. We have also fixed the maximum laser beam offset $(200 \mu \mathrm{m})$ to machine holes with diameters around $500 \mu \mathrm{m}$. Bearing this in mind, we started the process either at the periphery or at the center of the crater, so that the laser spot followed respectively an inwards spiral path or an outwards one by decreasing/increasing the beam offset during the process. In addition, we have checked the effect of processing with a single pass or two passes. The number of passes and the trend of beam offset variation play a significant role in the debris accumulation processes on the hole bottom as we will show in this work. Concerning the laser parameters, the pulse energies ranged from 0.1 to $0.4 \mathrm{~mJ}$. Pulse energy is the main parameter which determines the amount of material removed per laser pulse and therefore the hole depth. In this case, we need very high energies since the volume of enamel to be removed is very large.

In all cases, processing was carried out in air, without a water coolant.

\section{Analysis of samples}

\section{Light microscopy}

All samples were examined with an Axio M1 (Carl Zeiss, Germany) light microscope, operating in the dark-field mode. We used Epiplan $\times 20$ and $\times 50$ HD objectives (Carl Zeiss Vision), attached to a 1,300 $\times 1,030$-pixel digital camera (AxioCam HR, Carl Zeiss Vision). The images were processed with AxioVision software. This software permits the measurement of the depth and diameter of the cavities.

\section{Scanning electron microscopy}

A ZEISS DSM 940 scanning electron microscope was used to obtain images of the processed areas of the samples at different levels of magnification.

\section{Results}

A qualitative result of the effects of two different techniques for cavity preparation was observed in this study: on one hand, with a femtosecond laser at $795 \mathrm{~nm}$, and on the other, with the conventional mechanical technique. The procedure was observed and analyzed using light and SEM. The SEM images reveal the ability of the femtolaser to produce highprecision cavities in tooth enamel. No signs of collateral damage or cracks were observed, unlike with the conventional mechanical technique.

The light microscopy images point to the irregularity of the cavities made with conventional mechanical instrumentation and the perfect cavity boundary defined in those made with the femtosecond laser.

Figure 1 shows several rectangular cavities made with the femtosecond laser in enamel. The cavities were ablated using different pulse energies: $0.05 \mathrm{~mJ}$ (row A), $0.03 \mathrm{~mJ}$ (line B), $0.01 \mathrm{~mJ}$ (row C), and finally, $0.007 \mathrm{~mJ}$ (row D). Essentially, the greater the energy of the pulse, the deeper the microstructure. The pitches between the meander lines were $0.02 \mathrm{~mm}$ (column 1) and $0.01 \mathrm{~mm}$ (column 2). Pitch determines the overlapping of the ablated areas. Since the spot size is constant on the enamel surface for the same pulse energy, over-large pitches give rise to inhomogeneous ablation if the spot size is smaller than the pitch. This was the case of the structures in column 1 for both pulse energies, and for case $\mathrm{D}$ in column 2, where the pulse energy was very low. In all cases the scanning velocity was $0.05 \mathrm{~mm} / \mathrm{s}$.

Figure 2 shows magnified SEM images corresponding to the lower two energies, both pitches and the same scanning velocity as used in Fig. 1. Focusing on Fig. 2a ( $E=0.007 \mathrm{~mJ}$, $v=0.05 \mathrm{~mm} / \mathrm{s}$ and $s=0.01 \mathrm{~mm}$ ), the processed area is seen to have resulted in a shallow square cavity of approximately $215 \mu \mathrm{m}$ per side, with a homogeneous bottom. There are no signs of heat damage or cracks on the surrounding or bottom

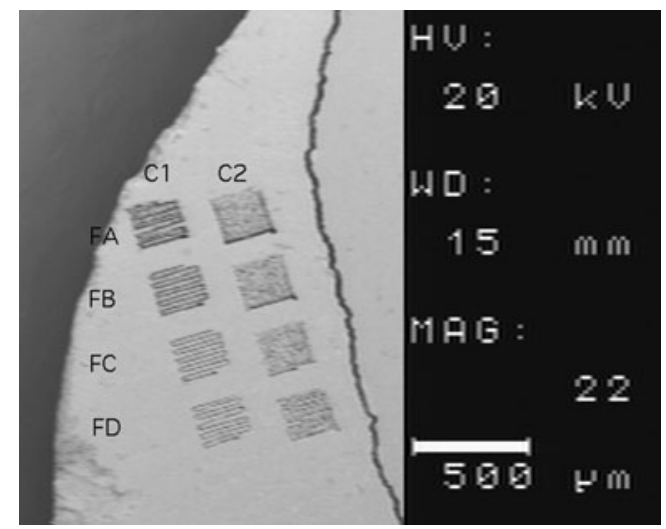

Fig. 1 SEM micrograph of cavities in enamel performed with the femtosecond laser (original magnification: $S E M \times 374$ ) 
surfaces. In the work described in Fig. 2b, we used the same parameters except for the pitch $(s=0.02 \mathrm{~mm})$. This time the squared cavity was $230 \mu \mathrm{m}$ per side, but the ablated and intact surfaces alternated as a result of the large degree of pitch as compared to the spot size, which was of the order of $0.01 \mathrm{~mm}$. The results obtained upon increasing the pulse energy $(E=0.05 \mathrm{~mJ})$ are shown in Fig. $2 \mathrm{c}$ and d. The square cavity was approximately $225 \mu \mathrm{m}$ per side for the smallest pitch (Fig. 2c), and $215 \mu \mathrm{m}$ for the largest one (Fig. 2d). In comparison with Fig. $2 \mathrm{a}$ and $\mathrm{b}$, the cavities were now much deeper since the ablation rate increased with the pulse energy. The effect of a pitch larger than the spot size is still visible in the case of Fig. 2d, although to a lesser extent since the spot size was now larger (close to $0.02 \mathrm{~mm}$ ).

Figure 3(a, b, c, d) shows the SEM and light microscopy images comparing the results of the two different treatments used in this study: $(a, c)$ the tooth surface irradiated by a femtosecond laser with SEM; (b, d) abrasion surface with a conventional diamond bur with light microscopy. The abrasion surface with a conventional diamond bur at a $v=450,000 \mathrm{rpm}$ under a water coolant (Fig. 3b) has an irregular surface. The smear layer is present on the surface of the cavity. The enamel rods are completely obliterated by the fusion of material due to the thermal effect.

The cavity prepared with the femtosecond laser (Fig. 3a) has a diameter of $500 \mu \mathrm{m}$ and the cavity boundary is perfectly defined on both the surface and on the walls. Some of the material is seen to have been redeposited at the bottom of the cavity. The debris is driven towards the previously ablated areas. Therefore, if the sample motion finishes at the boundary - as is the case in Fig. 3a-the debris are driven to the center of the cavity, whereas it is driven towards the boundary if the motion finishes at the center (Fig. 3c). The use of different sets of parameters - including the inwardoutward paths - did not solve this issue.

Figure $3 \mathrm{~d}$ shows detailed light microscope images of a cavity made with the turbine drill at $v=450,000 \mathrm{rpm}$. Unlike the cavities made with the femtosecond laser, in this image it is possible to observe the presence of microcracks around the cavity. This is a product of the mechanical and thermal effects that occur with conventional mechanical instrumentation. This is not desirable because microorganisms can penetrate through these microcracks, favoring failure of the restoration and the possible start of new caries.

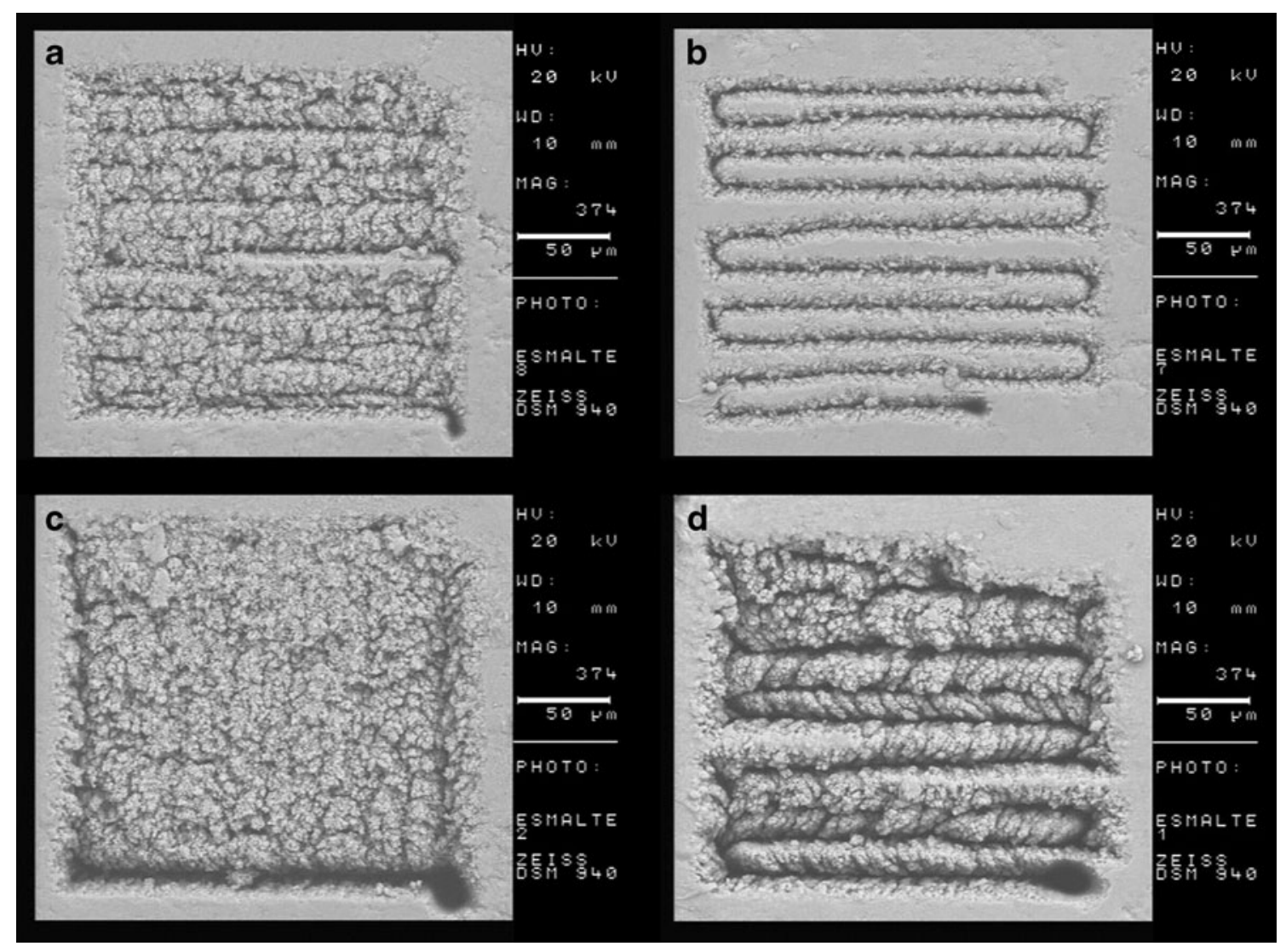

Fig. 2 SEM micrograph of femtosecond laser cavities in enamel with: a $E=0.007 \mathrm{~mJ}, v=0.05 \mathrm{~mm} / \mathrm{s}$ and $s=0.01 \mathrm{~mm}$ (original magnification, $\mathrm{SEM} \times 374$ ); b $E=0.007 \mathrm{~mJ}, v=0.05 \mathrm{~mm} / \mathrm{s}$ and $s=0.02 \mathrm{~mm}$ (original magnification, $\mathrm{SEM} \times 374)$; $\quad E=0.05 \mathrm{~mJ}, v=0.05 \mathrm{~mm} / \mathrm{s}$ and $s=$
$0.01 \mathrm{~mm}$ (original magnification, SEM $\times 374$ ); $\mathbf{d}$ SEM micrograph of femtosecond laser cavities in enamel with $E=0.05 \mathrm{~mJ}, v=0.05 \mathrm{~mm} / \mathrm{s}$ and $s=0.02 \mathrm{~mm}$ (original magnification, SEM $\times 374$ ) 


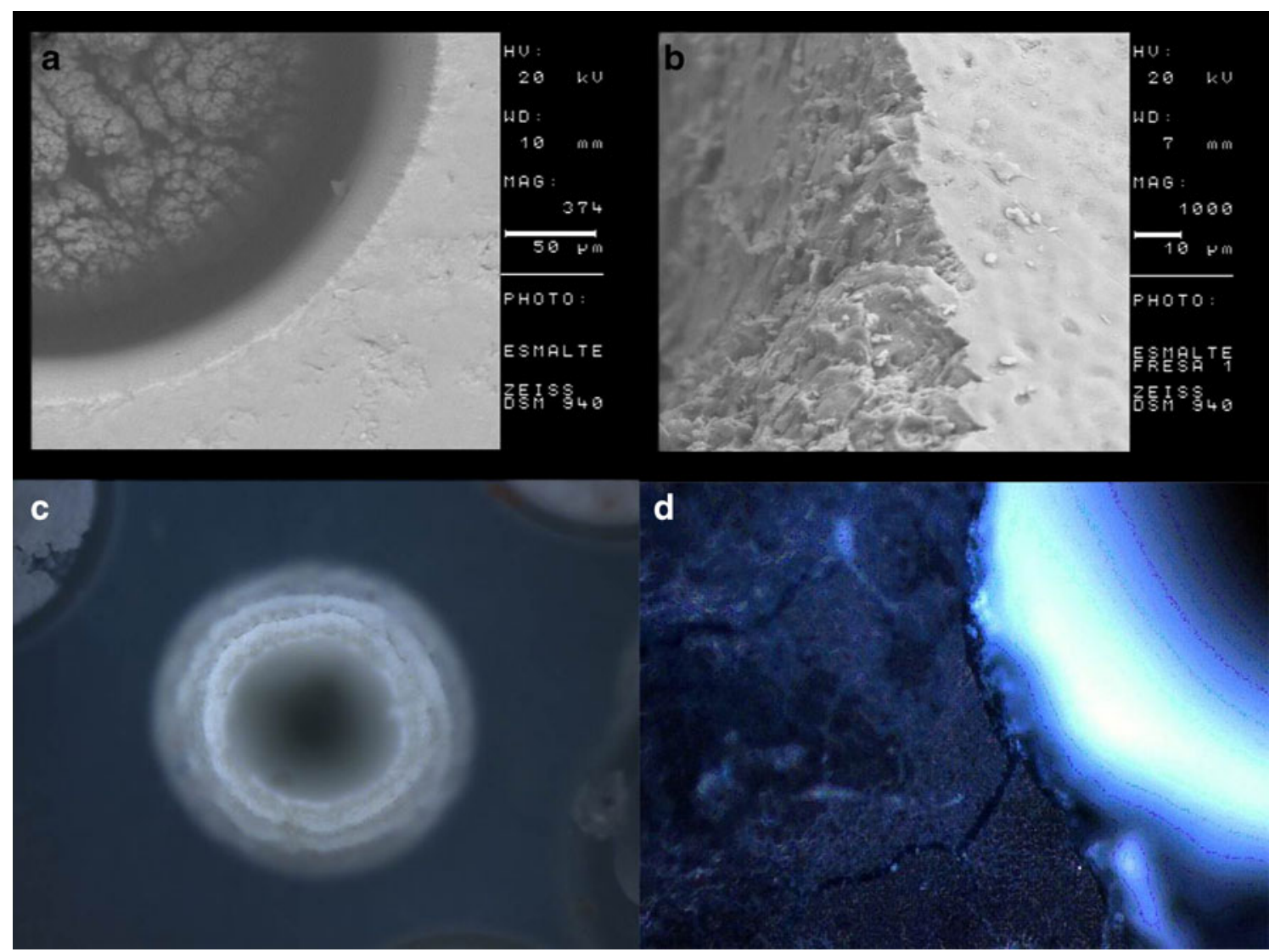

Fig. 3 a SEM micrograph of a circular cavity in enamel induced by femtosecond laser ablation (original magnification, SEM $\times 200$ ). The laser spot was initially located at the periphery and the beam offset was reduced to zero and again increased to its maximum (two passes). The pulse energy was set to $0.4 \mathrm{~mJ}$; b SEM micrograph of mechanical instrumentation cavities in enamel at a speed of 450,000 rpm under abundant water coolant (Boundary of the cavity; original

\section{Discussion}

Many studies have focused on the application of ultrashortpulsed lasers on hard dental tissues [11, 20-22]; however, few of them have addressed their application to the enamel exhaustively [10]. The suppression of microcracks with ultrashort pulses is of great importance in dentistry because these may become the source of new caries. In the present study, we used a femtosecond laser for the ablation of human enamel with the aim of minimizing these microcracks, which systematically appear with conventional mechanical techniques. Conventional techniques for drilling enamel lead to the formation of microfractures on the surface as a result of the high thermal and mechanical loads. In addition, melting and resolidification give rise to a smear layer on the walls of the cavities, such that further treatments are required to provide optimal access of restorative materials to them [23].

The SEM images reveal the ability of the femtolaser to produce high-precision cavities in tooth enamel magnification, SEM $\times 500$ ); $\mathbf{c}$ cavity performed by means of femtosecond laser ablation. The laser spot was initially located at the center and the beam offset was increased to its maximum and back to zero (two passes). The pulse energy was set to $0.4 \mathrm{~mJ}$; $\mathbf{d}$ presence of cracks in a cavity made with an air-turbine at a speed of 450,000 rpm under abundant water coolant

(Figs. 2a-d, 3a). No signs of collateral damage and cracks were observed, unlike with conventional milling. As described previously in the literature for other biological tissues, the application of subpicosecond pulses almost completely eliminates thermal damage and the appearance of microcracks in adjacent areas $[24,25]$. Rode et al. concluded that the removal of enamel using a subpicosecond laser allows the side effects produced by air turbines to be eliminated. Moreover, this technique permits the painless removal of hard dental tissue, thereby enhancing the efficiency of clinical procedures [26, 27] (Figs. 1, 2a-c, 3a).

We tested different geometries and processing parameters. Our conclusion is that careful selection of the parameters is crucial for achieving precise and high-quality structures on the enamel surfaces. The pulse energy determines the rate of material removal, but very high energies tightly focused on the surface could lead to some damage since phase explosion mechanisms begin to come into play. The scanning speed determines the number of pulses contributing to ablation in a given region of the surface. The 
faster the scan, the lower number of pulses, and hence lower ablation rates. Both parameters decisively influence the depth of the cavity. The morphology is driven by the sample motion features. The dimensions of the cavities performed were not affected by these parameters, remaining approximately at $200-250 \mu \mathrm{m}$.

We tested two different cavity patterns with rectangular and circular shapes. A meandering and a spiral path were respectively used to produce these geometries. In both cases, the ratio between the pitch and the laser spot size on the surface was very important. A ratio above unity could lead to incomplete ablation of the cavity.

Finally, ablation debris is deposited at the bottom of the cavities. Its shape is the result of the rapid volume expansion of the evaporating water contained in the tissue. The location of the debris is determined by the scanning procedure. We checked the possibility of eliminating the debris without using any pressured fluid or gas. Also, we tested some different sample motion strategies, but without success.

According to the results obtained here, it may be stated that human tooth enamel can be successfully removed with femtosecond lasers, in agreement with other investigations addressing the ultrashort-pulsed laser ablation of hard dental tissue [25-27].

This system has many advantages over the mechanical technique and techniques using other types of laser. These are discussed below.

1. When mechanical instrumentation is used, friction generates high temperatures, which cause irreversible damage to the tooth; the tooth surface shows signs of thermal and mechanical damage, together with the presence of a smear layer that is formed after implementation of this technique. In contrast, the femtosecond (Ti/ Sapphire) laser causes minimal structural changes to the irradiated enamel. The surfaces show a complete lack of thermal or mechanical damage such as cracks, craters, or charring. The treated surface has a rough and irregular aspect without a smear layer or cracks. These benefits have been also described by several other authors [25].

2. Additionally, the size and shape of burs does not allow complex shapes or minimally invasive treatment [28]. The femtosecond laser affords the possibility of making incisions ten times smaller than that permitted by the smallest bur [29] and allows greater control of the removal of material.

3. Preparations with mechanical instrumentation require the accompanying use of irrigation to prevent pulp involvement after it has undergone the high temperatures generated inside the cavity. In addition, when using lasers of the erbium family on enamel, this requires the joint use of irrigation to reduce possible temperature increases. This irrigation tends to reduce the visibility of the operative field.

4. Ultrashort pulse laser ablation means that irrigation is not required because the process occurs in a shorter time, in which the materials do not have the necessary time to transmit heat (of the order of tens to hundreds of picoseconds), and the generation of a stable particle flux is not possible [22].

5. With the use of the femtosecond laser, patient stress would be theoretically reduced since there is no noise or need for anesthesia on performing the cavity, unlike the case of mechanical ablation.

6. Ultrashort pulse lasers enable the effective ablation of nearly all materials, including metal restorations, which cannot be removed with erbium lasers. This is because when performing femtolaser ablation, the absorption coefficient of the material depends on the intensity of the radiation rather than on the wavelength, as in the case of conventional lasers.

This study was an initial project whose results suggests that the application of ultrashort pulse lasers as contactless drills in dentistry may provide an alternative to classical mechanical techniques, aimed at improving the quality of dental treatment. Further studies will focus on the bonding of dental materials to laser-irradiated enamel.

\section{Conclusions}

In the present study, we demonstrate the ability of femtolasers to produce high-precision cavities in tooth enamel. The femtosecond laser is presented as an optimal tool for the treatment of dental decay and as an alternative to air-turbine drills to reduce the mechanical damage that occurs during the removal of hard dental tissue.

Acknowledgments A.G. and P.M. acknowledge the support of Spanish Ministerio de Ciencia e Innovación through the Consolider Program SAUUL (CSD2007-00013) and research project FIS200909522, from Junta de Castilla y León through the Program for Groups of Excellence (GR27) and of the EC Seventh Framework Programme (LASERLAB-EUROPE, grant agreement no. 228334). We also acknowledge the support of the Centro de Laseres Pulsados, CLPU, Salamanca, Spain.

\section{References}

1. Yaman BC, Guray BE, Dorter C, Gomeç Y, Yazıcıoglu O, Erdilek D (2011) Effect of the erbium:yttrium-aluminum-garnet laser or diamond bur cavity preparation on the marginal microleakage of class V cavities restored with different adhesives and composite systems. Lasers Med Sci 27(4):785-794

2. Anusavise KJ (1997) Efficacy of nonsurgical management of the initial caries lesion. J Dent Educ 6:895-905 
3. Peters M, Mclean I (2001) Minimal intervention and concepts for minimally invasive cavity preparations. J Adhes Dent 3:7-16

4. Colucci V, do Amaral FL, Pécora JD, Palma-Dibb RG, Corona SA (2009) Water flow on erbium:yttrium-aluminumgarnet laser irradiation: effects on dental tissues. Lasers Med Sci 24:811-818

5. Fornaini C, Riceputi D, Lupi-Pegurier L, Rocca JP (2012) Patient responses to Er:YAG laser when used for conservative dentistry. Lasers Med Sci (in press)

6. Keller U, Hibst R (1989) Experimental studies of the application of the Er:YAG laser on dental hard substances II. Light microscopic and SEM investigations. Lasers Surg Med 9:345-351

7. Parker S (2007) Surgical lasers and hard dental tissue. Br Dent J 202:445-454

8. Lustosa-Pereira AC, Pozza DH, Cunha A, Dedavid BA, Duarte-de Moraes JF, Gerhardt-de Oliveira M (2011) Analysis of the morphology and composition of tooth apices apicectomized using three different ablation techniques. Med Oral Patol Oral Cir Bucal $16: 225-230$

9. Firat E, Gurgan S, Gutknecht N (2012) Microtensile bond strength of an etch-and-rinse adhesive to enamel and dentin after Er:YAG laser pretreatment with different pulse durations. Lasers Med Sci 27:15-21

10. Ji L, Li L, Devlin H, Liu Z, Jiao J, Whitehead D (2011) Ti:sapphire femtosecond laser ablation of dental enamel, dentine, and cementum. Lasers Med Sci 27(1):197-204

11. Kim BM, Feit MD, Rubenchik AM, Joslin EJ, Celliers PM, Eichler J, Da Silva LB (2001) Influence of pulse duration on ultrashort laser pulse ablation of biological tissues. J Biomed Opt 6:332-338

12. Niemz MH, Kasenbacher A, Strassl M, Bäcker A, Beyerti A, Nickel D et al (2004) Tooth ablation using a CPA-free thin disk femtosecond laser system. Appl Phys B 79:269-271

13. Zhang N, Wang W, Zhu X, Liu J, Xu K, Huang P, Zhao J, Li R, Wang M (2011) Investigation of ultrashort pulse laser ablation of solid targets by measuring the ablation-generated momentum using a torsion pendulum. Opt Express 19:8870-8878

14. Pronko PP, Dutta SK, Squier SJ, Rudd JV, Du D, Mourou G (1995) Machining of sub-micron holes using a femtosecond laser at 800 nm. Opt Commun 114:106-110

15. Chichkov BN, Momma C, Nolte S, von Alvensleben F, Tünnermann A (1996) Femtosecond, picosecond and nanosecond laser ablation of solids. Appl Phys 63:109-115
16. Varel H, Ashkenasi D, Rosenfeld A, Wähmer M, Campbell EEB (1997) Micromachining of quartz with ultrashort laser pulses. Appl Phys A 65:367-373

17. Nolte S, Momma C, Jacobs H, Tunnermann A, Chichkov BN, Wellegehausen B, Welling H (1997) Ablation of metals by ultrashort laser pulses. J Optic Soc Am B 14:2716-2722

18. Mourou G, Strickland D (1985) Compression of amplified chirped optical pulses. Opt Commun 55:447-449

19. Vázquez de Aldana JR, Méndez C, Roso L, Moreno P (2005) Propagation of ablation channels with multiple femtosecond laser pulses in dielectrics: numerical simulations and experiments. J Phys D Appl Phys 38:2764-2768

20. Girard B, Yu D, Armstrong MR, Wilson BC, Clokie CM, Miller RJ (2007) Effects of femtosecond laser irradiation on osseous tissues. Lasers Surg Med 39:273-285

21. Portillo M, Lorenzo MC, Sánchez JM, Peix M, Albaladejo A, García A et al (2012) Morphological alterations in dentine after mechanical treatment and ultrashort pulse laser irradiation. Lasers Med Sci 27:53-58

22. Ji L, Li L, Devlin H, Liu Z, Jiao J, Whitehead D (2012) Ti:sapphire femtosecond laser ablation of dental enamel, dentine, and cementum. Lasers Med Sci 27:197-204

23. Ekworapoj P, Sdihu SK, McCabe JF (2007) Effect of different power parameters of Er, Cr:YSGG laser on human dentine. Lasers Med Sci 22:175-182

24. Kohns P, Zhou P, Stormann R (1997) Effective laser ablation of enamel and dentine without thermal side effects. J Laser Appl 9:171-174

25. Pike P, Parigger C, Splinter R, Lockhart P (2007) Temperature distribution in dental tissue after interaction with femtosecond laser pulses. Appl Opt 46:8374-8378

26. Rode AV, Gamaly EG, Luther-Davies B (2002) Subpicosecond laser ablation of dental enamel. J Appl Phys 92:2153-2158

27. Rode AV, Gamaly EG, Luther-Davies B, Taylor B, Graessel TM, Dawes JM et al (2003) Precision ablation of dental enamel using a subpicosecond pulsed laser. Aust Dent J 48:233-239

28. Wieger V, Zoppel S, Wintner E (2007) Ultrashort pulse laser osteotomy. Laser Phys 17:438-442

29. Girard B, Cloutier M, Wilson DJ, Clokie CMI, Miller RJD, Wilson BC (2007) Microtomographic analysis of healing of femtosecond laser bone calvaria wounds compared to mechanical instruments in mice with and without application of BMP-7. Lasers Surg Med $39: 458-467$ 